\title{
Schulleitungen und evidenzbasierte Bildungsreform im Schulwesen
}

\author{
Herbert Altrichter (D) David Kemethofer • Ann Cathrice George
}

Angenommen: 4. Dezember 2018 / Online publiziert: 12. Dezember 2018

(C) Der/die Autor(en) 2018

Zusammenfassung In den deutschsprachigen Schulsystemen sind in den letzten Jahren neue, sog. „outputorientierte“ oder „,evidenzbasierte“ Steuerungskonzepte eingeführt bzw. akzentuiert worden, die mit veränderten Arbeitsanforderungen für Schulleitungen verbunden sind. An einem Sample von österreichischen Schulleitungen wird untersucht, ob es relevante Gruppen von Leitungspersonen gibt, die diese Steuerungsvorstellungen teilen, und ob entsprechende Einstellungen auch mit vermehrter Entwicklungsaktivität und einem veränderten Führungsverständnis zusammenhängen. Mittels Clusteranalyse konnten drei in Hinblick auf konkurrierende Steuerungsvorstellungen gut interpretierbare Gruppen identifiziert werden. Schulleitungen mit „outputorientierten Einstellungen“ berichten über mehr (unterrichtsbezogene) Entwicklungsaktivitäten und verstehen sich eher als Manager eines pädagogischen Betriebs.

Schlüsselwörter Schulleitung · Governance · Einstellungen · Schulleitungstätigkeit · Outputorientierung · Evidenzbasierung

\section{School leaders and evidence-based reform policies in education}

\begin{abstract}
German speaking countries have seen major changes in the governance of their school systems towards an output-oriented or evidence-based type of governance. As a consequence, rights, responsibilities, and tasks for school leaders have changed considerably. Based on a sample of Austrian principals we investigated whether there are distinctive groups of school leaders with respect to their governance-related attitudes and if there is a correlation between these attitudes and development activities and their conception of school leadership. A cluster analysis
\end{abstract}

H. Altrichter $(\square)$

Johannes Kepler Universität, Linz, Österreich

E-Mail: herbert.altrichter@jku.at 
identified three different groups of principals. Those school leaders who are particularly positive about output-oriented governance report more (teaching-related) development activities and interpret their role as managers of an educational enterprise.

Keywords School leadership · Governance · Attitude · Output-orientation · Evidence-based governance

\section{Einleitung: Bildungspolitischer und theoretischer Hintergrund}

\subsection{Governance-Reformen}

In den letzten zwanzig Jahren haben die deutschsprachigen Schulsysteme eine deutliche Veränderung ihrer „Governance“ erfahren (Martens et al. 2010; Altrichter und Maag Merki 2016). Unter Governance verstehen wir die Art und die Prinzipien, wie Entscheidungen zur Gestaltung und Weiterentwicklung des Bildungswesens auf seinen verschiedenen Ebenen fallen und wie die Akteure ihre Handlungen koordinieren (Schimank 2007; Altrichter 2015). Governance-Reformen streben an, die Entscheidungs- und Koordinationsgesichtspunkte im Bildungswesen zu verändern.

Der Governance-Wandel der letzten zwanzig Jahre in den deutschsprachigen Schulsystemen ist oft als eine (Analysegesichtspunkt 1=A1) Entwicklung von der „Input-“ zur „Outputorientierung“ (vgl. Fend 2011; Kuper 2008; Zukunftskommission 2005) sowie als (A2) eine (partielle) Veränderung der Aufgaben und des Zusammenspiels verschiedener Ebenen und Akteure im Bildungswesen (Altrichter und Maag Merki 2016) beschrieben worden. Wir werden diese „Schlüsselelemente der Reform“ (Brauckmann und Pashiardis 2012, S. 21) weiter unten zur Beschreibung von bildungspolitischen Entwicklungen verwenden.

Unter einer eher ,input-orientierten Governance“ versteht man ein solches Bildungssystem, bei dem wesentliche Entscheidungen vor den Arbeits- und Unterrichtsprozessen in einer Schule fallen. Durch die Festlegung von relativ genauen Regeln (Gesetze, Verordnungen, regionale Verhaltensnormen) auf den den Einzelschulen vorgelagerten Ebenen (Parlament/Bildungspolitik, Ministerium, regionale Administration) und durch die zentral/regional gesteuerte Vergabe von Ressourcen (Lehrerstunden, Approbation/Vergabe von Lehrbüchern und Unterrichtsmitteln u.v.m.) soll die Qualität schulischer Tätigkeit sichergestellt werden. Ein solches Steuerungsmodell beruht im Wesentlichen auf einer ,logic of confidence and good faith“ (Meyer und Rowan 1977, S. 357). In einem ,output-orientierten Steuerungssystem“ sollen dagegen wesentliche Entwicklungsentscheidungen angesichts der tatsächlich erzielten Ergebnisse (also nach den Arbeits- und Unterrichtsprozessen) gefällt werden. Dazu ist es notwendig, dass die Ergebnisziele (z. B. durch Bildungsstandards oder Qualitätsziele für Schulinspektionen) explizit gemacht werden, dass die Erreichung dieser Ziele gemessen wird (z. B. durch Lernstandserhebungen und Schulinspektionen) und dass die (ev. beobachteten) Ist-Soll-Diskrepanzen als Datenfeedback an die Akteure auf verschiedenen Ebenen des Schulsystems kommuniziert 
werden. Von diesen wird erwartet, dass sie die Informationen für ,rationale Entwicklungsentscheidungen“ nutzen.

Altrichter et al. (2005) haben dabei drei Phasen dieser Transformation der Systemsteuerung unterschieden: Um die Mitte der 1990er Jahre wurden - unter dem Schlagwort Schulautonomie - vermehrte Entscheidungsspielräume an den Einzelschulen geschaffen. Schulleitungen und Lehrpersonen sollten mehr Verantwortung übernehmen und für Entwicklungsanstrengungen motiviert werden. Dadurch sollte die Qualität und lokale Responsivität der Entscheidungen erhöht werden.

Um die oben genannten Analysegesichtspunkte aufzunehmen: Offenbar wird in dieser Phase (A1) die Steuerung schulischer Tätigkeit in Form genauer Definition des normativen „Inputs“ etwas zurückgenommen (später wird auch die Vor-Steuerung der Ressourcen etwas gelockert, indem Schulen z. B. durch Sponsoring, die Vermietung von Schulräumlichkeiten und den Verkauf von schulischen Leistungen zusätzliche Einnahmen erlaubt werden). (A2) Dadurch wird der Akteur Einzelschule (und die dort tätigen Lehrpersonen) relativ bedeutsamer, während die Schulaufsichtsbeamten als Interpreten und Kontrolleure vorgegebener Regeln potentiell an Bedeutung verlieren. Die Idee der Einzelschulentwicklung, die schon seit den 1980er Jahren eine gewisse Orientierung für schulische Tätigkeit bot (Fend 1986), wurde dadurch propagiert und strukturell durch Schulautonomie gestärkt.

Eine zweite Phase in der zweiten Hälfte der 1990er Jahre ist durch eine Aufwertung der Schulleitung sowie neue Aufgaben für die Schuladministration charakterisiert. Einesteils wurde die innerschulische Führung durch Einführung neuer Koordinationsinstrumente (wie Schulprogramme, Selbstevaluation, innerschulisches Qualitätsmanagement) gestärkt, anderenteils sollten schulübergreifende Vorgaben den „,autonomeren“ Einzelschulen wünschenswerte Ziele der Entwicklung signalisieren (z.B. durch Aufgabenbeispiele, Vergleichsarbeiten, „Qualitätstableaus“).

Gemäß den oben eingeführten Analysegesichtspunkten scheint diese Phase vor allem durch die (A2) Aufwertung der Schulleitungen charakterisiert; sekundär sucht die Schuladministration partiell neue Aufgaben, die in der Formulierung und Propagierung von schulübergreifenden Zielvorgaben bestehen. (A1) Andererseits könnte diese Phase auch als Vorbereitung und ,sanfte“ Gewöhnung an outputorientierte Praktiken interpretiert werden: Schulübergreifende Ziele, Schulprogramme und Qualitätsmanagement machen mit Zielvorgaben und Ergebnisevaluationen vertraut.

Eine dritte Phase der Governance-Modernisierung wurde durch die für die deutschsprachigen Schulsysteme unerwartet schlechten Ergebnisse bei PISA 2000 und den daraus entstandenen medialen Druck stimuliert und geprägt. Bildungsstandards sollten die Ergebnisse schulischen Lernens in einer messbaren Kompetenzsprache beschreiben, Qualitätstableaus zusätzlich Prozessqualitäten klarmachen. Durch vergleichende Lernstandserhebungen und „,neue Schulinspektionen“ wurde die Erreichung dieser Ziele kontrolliert, in Berichten rückgemeldet und als Impuls für Qualitätsentwicklung verstanden. 
In dieser Phase werden also (A1) Instrumente zur Umsetzung von Outputorientierung (oft auch als evidenzbasierte Steuerung ${ }^{1}$ bezeichnet) ausgearbeitet, auf „ganzer Breite“ bereitgestellt und als wesentliche Elemente des Entscheidens und Entwickelns auf allen Systemebenen postuliert. (A2) Auf der Ebene der Akteure ist die Situation weniger einfach zu beschreiben als in den Phasen zuvor, weil nun grundlegendere Umorientierungen von Aufgaben und Verantwortlichkeiten angestrebt werden. Die Berufstätigen in den Einzelschulen sollen weiterhin Energien in ,autonome Schulentwicklung“ kanalisieren, diese jedoch - in einer Autonomie der Wege, nicht der Ziele (Heid 2003) - stärker auf die vorgegebenen Kompetenz- und Prozessziele ausrichten. Schulleitungen werden noch bedeutsamer, weil sie - damit zielgerichtete und ergebnisorientierte Entwicklung geschieht - die Lehrpersonen zur Beachtung von Zielen, zur Interpretation von Datenfeedback und zu unterrichtsbezogenen Entwicklungshandlungen motivieren, koordinieren und unterstützen müssen. Auch die Schulaufsicht wird deutlich aktiver konzipiert: Beispielsweise soll sie im österreichischen Qualitätsmanagementsystem „Schulqualität Allgemeinbildung“ (SQA bzw. dem Äquivalent in den berufsbildenden Schulen QIBB; vgl. Altrichter 2017) in „Bilanz- und Zielvereinbarungsgesprächen“ die Einzelschulen motivieren, - nicht zuletzt durch die Standardüberprüfungen entdeckte - Entwicklungsprobleme in Angriff zu nehmen, selbst regionale Entwicklung unterstützen und überregionale Bedarfe an übergeordnete Ebenen kommunizieren.

\subsection{Schulleitungen als gate keepers und Führungspersonen für Bildungsreformen}

Die Forcierung outputorientierter Steuerungskonzepte hat die Rahmenbedingungen professionellen Führungshandelns verändert und zu gestiegenen Anforderung an Schulleitung geführt (wie auch der zweite Analysegesichtspunkt oben gezeigt hat). Ergänzend zu administrativen und pädagogischen Tätigkeiten sind Schulleitungen in einer neuen Steuerungslandschaft dazu angehalten, zielgerichtete und systematische Entwicklungen zu betreiben und Rechenschaft darüber abzulegen. Dadurch ergeben sich neue Verantwortlichkeiten und ein geändertes Aufgabenprofil (Brauckmann und Pashiardis 2016). Dazu zählen insbesondere Aufgaben von Schul- und Qualitätsmanagement, Öffentlichkeitsarbeit sowie Schul-, Unterrichts- und Personalentwicklung (Schratz 2016). So sorgt eine erfolgreiche Schulleitung auch für unterrichtsbezogenes Feedback, gezielte Lehrerfortbildung und Nutzung von internen wie externen Evaluationsdaten, um evidenzbasierte Weiterentwicklung zu unterstützen. Darüber hinaus schafft sie Visionen und Ziele für die Schule und kümmert sich um Reformbelange (Brauckmann und Herrmann 2012). Manche Beobachter/innen vermuten Interferenzen zwischen neuen Anforderungen und bestehendem Aufgabenverständnis und in der Folge Identitäts-, Legitimitäts-, Rollen- und Funktionskonflikte. Auf der anderen Seite werden gerade die Schulleitungen als ,gate keepers“ angesehen, deren Führungshandeln entscheidend dazu beiträgt, ob die neuen bildungspolitischen

\footnotetext{
1 Die Problematik dieses Begriffs kann hier nicht weiter diskutiert werden (vgl. Jornitz 2008; Bellmann 2016). Wir verwenden im Folgenden Outputorientierung und Evidenzbasierung synonym.
} 
Konzepte an den Schulstandorten zum Leben kommen (Brauckmann und Schwarz 2015).

Schulleitungen sollen Prozesse der „Rekontextualisierung“ (Fend 2006) am Schulstandort stimulieren, organisieren und koordinieren, durch die die Strukturangebote der neuen Steuerungsansätze in Arbeitspraktiken und -strukturen, die den Reformintentionen entsprechen, übersetzt werden (Ball et al. 2012). Dafür müssen Schulleitungen diese Intentionen verstehen und praktische Ideen für die Transformation der Reformelemente in die alltägliche Arbeit haben (Spillane 2012).

Sowohl in Politik als auch Forschung besteht Konsens darüber, dass Schulleitungen maßgeblich für die Qualität einer Schule verantwortlich sind. Ihr Führungsverhalten beeinflusst Schulorganisation, Schulkultur, Lehr-Lern-Arrangements und damit in letzter Konsequenz die Leistung von Schüler/innen (z. B. Day et al. 2016; Hallinger 2011). Schulleitungen, die diese Reformen umsetzen wollen, werden selbst ein evidenzbasiertes Schulleitungshandeln zeigen, das heißt einerseits die durch die Monitoring-Aktivitäten bereitgestellten Informationen rezipieren und andererseits selbst durch Schulevaluation und Beobachtung des Umfeldes Daten suchen. Sie werden die verfügbaren Informationen interpretieren, in Entwicklungsentscheidungen umsetzen, Implementationsprozesse begleitend auswerten sowie Lehrpersonen motivieren, in gleicher Weise zu handeln. Letztlich müsste sich ein solches Schulleitungshandeln in mehr und gezielteren Entwicklungsprozessen und in gesteigerter Schulqualität niederschlagen.

Die empirische Schulforschung hat sich jüngst dem Schulleitungshandeln im Kontext von evidenzbasierter Bildungsreform zugewandt (Mintrop und Coghlan 2018): Brown und Zhang (2017) fanden einen positiven Zusammenhang zwischen dem gezielten Einsatz von Evidenzen und der Leistungsfähigkeit von Lehrpersonen und Schulen. Gleichzeitig wiesen Earl und Fullan (2003) auf die Herausforderungen effektiver Datennutzung hin und beschrieben die Hoffnungen und Bedenken von Schulleitungen gegenüber evidenzbasierten Reformen.

Ein differenzierter Blick zeigt, dass es unterschiedliche Typen im Hinblick auf Datennutzung gibt. Stamm (2003; ähnlich Demski 2017; Groß Ophoff 2013) konnte vier Muster identifizieren: Blockade, Innovation, Alibi und Reaktion, wobei lediglich in den Gruppen Innovation und Reaktion eine direkte Nutzung von Evaluationsinformationen erfolgte. Wurster und Gärtner (2013) fanden fünf Muster des Umgangs mit Inspektionsergebnissen. „Aktive“ und ,aktiv unzufriedene Schulen“ zeigten hohes Aktivitätsniveau und nahmen externe Unterstützung in Anspruch. ,,(Selbst-)zufriedene“, ,reaktive“ und ,,passiv unzufriedene“ Schulen waren hingegen aus unterschiedlichen Gründen wenig aktiv. Eine Klassifizierung von Schulleiter/innen hinsichtlich ihres Managements von Schulautonomie und bildungspolitischen Veränderungen ergab vier Leitungstypen, die als zuversichtliche, vorsichtige, besorgte und durch staatliche Anforderungen eingeschränkte Schulen eingestuft wurden (Higham und Earley 2013).

Eine Erklärung für die unterschiedliche Datennutzung könnten die Arbeiten von Huber et al. (2013) bzw. Brauckmann und Schwarz (2015) liefern. Brauckmann und Schwarz (2015) fanden, dass Schulleitungen für jene Tätigkeiten, die sie als wichtig einschätzen, auch durchschnittlich mehr Zeit verwenden als jene, die diese Aufgaben nicht als wichtig empfinden. Allerdings spiegeln sich jene Aufgaben, die als hoch 
bedeutsam eingeschätzt werden, nicht im täglichen Arbeitspensum wider, weil die meiste Arbeitszeit für Unterricht und Administration verwendet wird. Insbesondere administrative Tätigkeiten (wie Berichte verfassen, Akten sichten, Schulstatistik führen) oder auch das Umsetzen von Schulreformen werden von Schulleiter/innen als die im Vergleich zu anderen Aufgaben am stärksten belastenden Tätigkeiten wahrgenommen (Huber et al. 2013). Kronsfoth et al. (2018) fanden in einer explorativen Schulleitungsbefragung ein ,insgesamt eher diffuses Bild“. Tendenziell stellen sie einige Zusammenhänge zwischen Führungskonzepten und Datennutzung zur Diskussion: Eine ,delegative Führung“, die Datennutzung und Entwicklungsaufgaben an Lehrpersonen weiterreicht, korreliert mit weniger Datennutzung durch Schulleitung und Lehrkräfte und trägt dadurch möglicherweise zu einer ,Verantwortungsdiffusion“ bzgl. Datennutzung bei. „Direktive Schulleitungen“ scheinen dagegen auch klare Vorschriften für Datennutzung zu geben, was mit einer ,relativ intensiven Rezeption“ solcher Informationen einhergeht (Kronsfoth et al. 2018, S. $58 \mathrm{f}$.).

\subsection{Governancebezogene Einstellungen und Reformaktivitäten}

Als Bedingungen für wirksames Schulleitungshandeln hat Hallinger (2011) neben Wissen und Erfahrung insbesondere Überzeugungen, Werte und Einstellungen genannt, die Entscheidungen leiten und sich so auf Prozesse und Abläufe in der Schule auswirken. Auch wenn Einstellungen nicht mit realisierten Handlungen in eins gesetzt werden dürfen (Ajzen 1991), können positive Einstellungen zu Outputorientierung und Evidenzbasierung als eine notwendige Voraussetzung für Führungshandeln, das den Intentionen der Reform entspricht, angesehen werden. So wird eine positive Einstellung zu einem Reformvorhaben (Akzeptanz) sowohl in allgemeinarbeitsbezogenen Kontexten (Wanberg und Banas 2000) als auch im Zusammenhang mit Bildungsreformen als eine günstige Voraussetzung für das Engagement der Berufstätigen bei der Implementation der Neuerung angesehen. Tatsächlich besteht ein positiver Zusammenhang zwischen Einstellung zur Datenrückmeldung und Reflexionsprozessen und Entwicklungsaktivitäten (Groß Ophoff 2013; Wiesner et al. 2016; Zeitler et al. 2013).

Schulleitungen werden häufig nach Bewertungen von Reformideen befragt. Uns interessiert hier aber nicht so sehr die Akzeptanz von spezifischen Innovationsideen, sondern wie Schulleitungen zu den hier diskutierten großflächigen Veränderungen im Steuerungssystem stehen, ob sie bereit sind, diese in ihrer Leitungstätigkeit mitzutragen, und ob sich diese auf ihre Leitungshandlungen auswirken. In verschiedenen Studien (Altrichter et al. 2012a; Altrichter und Kemethofer 2015; Kemethofer und Altrichter 2017) wurde die Bewertung des Potentials verschiedener Steuerungsinstrumente und -strategien für die Entwicklung der Qualität des österreichischen Schulwesens durch österreichische Schulleitungen erfragt. Dabei zeigte sich eine steuerungstheoretisch gut interpretierbare - Faktorenstruktur, in der fünf steuerungsbezogene Einstellungen zur Qualitätsentwicklung im Schulwesen (vgl. auch Abb. 1) unterscheidbar sind: 


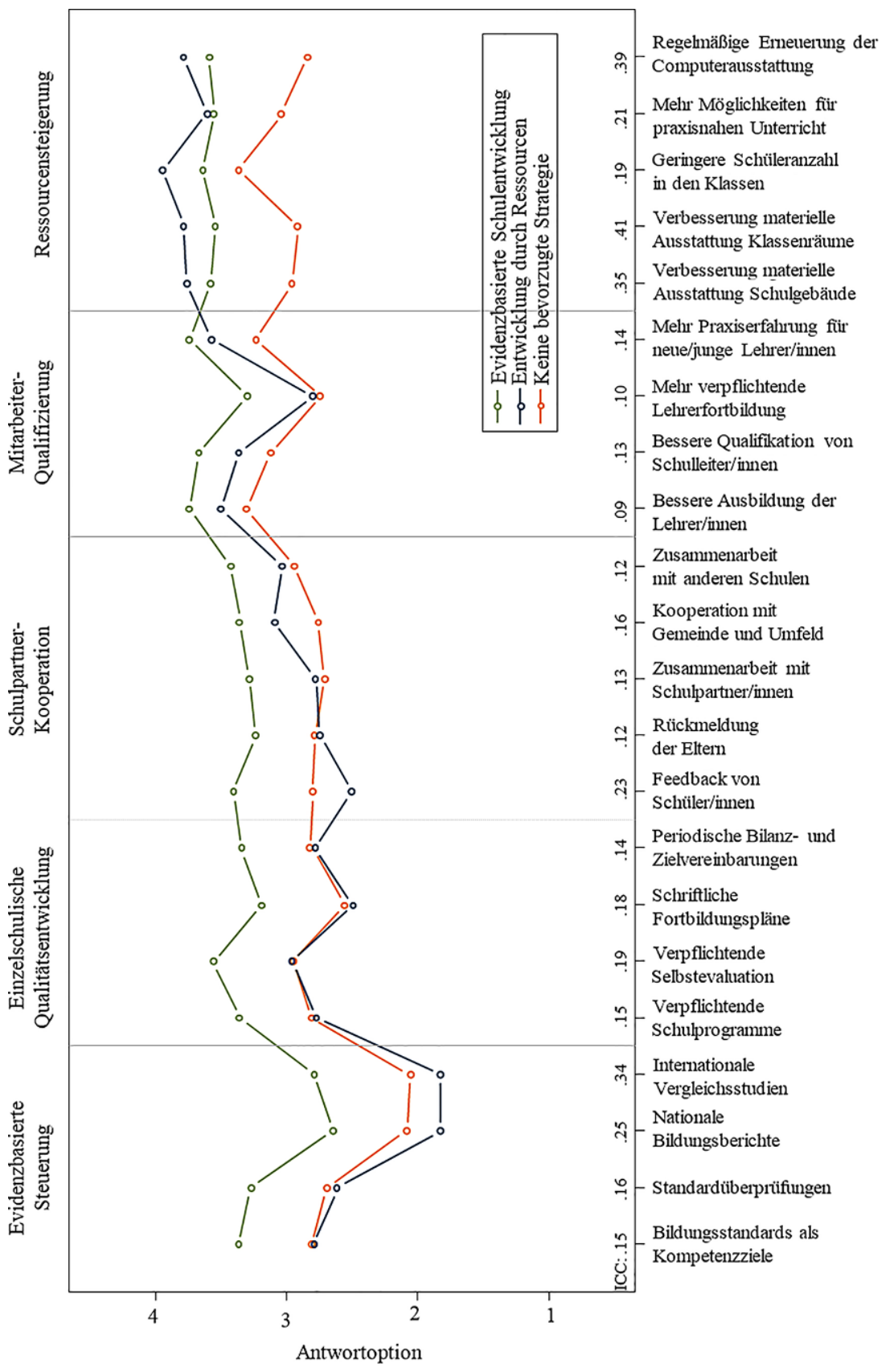


a) Ressourcensteigerung umfasst Vorstellungen zur Qualitätssteigerung durch Senkung der Klassenschülerzahl sowie durch Verbesserung der Computerausstattung sowie der materiellen Ausstattung von Klassenräumen und Schulgebäuden

b) Mitarbeiter-Qualifizierung umfasst Vorstellungen zur Qualitätssteigerung durch bessere Lehrer- und Schulleiterausbildung, durch mehr Praxiserfahrung für neue Lehrer/innen sowie durch mehr verpflichtende Lehrerfortbildung

c) Schulpartner-Kooperationen umfasst Vorstellungen zur Qualitätssteigerung durch Zusammenarbeit mit Schulpartner/innen, durch Einholung von Eltern- und Schülerfeedback sowie durch Zusammenarbeit mit anderen Schulen, Gemeinde und Umfeld

d) Einzelschulische Qualitätsinstrumente umfasst Vorstellungen zur Qualitätssteigerung durch innerschulische Steuerungs- und Entwicklungsinstrumente (Selbstevaluationen, Schulprogramme und schriftliche Fortbildungspläne), wobei deren Verpflichtungscharakter und jährliche Leistungsvereinbarung mit der Schulaufsicht nicht gescheut werden

e) Evidenzbasierte Steuerung umfasst schließlich Vorstellungen zur Qualitätssteigerung durch Bildungsstandards, standardbezogene Tests, Nationale Bildungsberichte und die Ergebnisse internationaler Vergleichsstudien

Diese fünf Einstellungsbündel lassen sich zur Diskussion über Veränderungen der Systemsteuerung in Abschn. 1.1 in Beziehung setzen. Die ersten beiden Faktoren betreffen materielle (Faktor a) und personelle (Faktor b) Ressourcen, die üblicherweise den Input-Faktoren zugerechnet werden. In beiden Fällen sind dies Entscheidungen, die in einem bürokratisch-zentralistischen Governance-System fallen, bevor die Schule tätig wird, und daher weitgehend dem Einfluss der Schulleitungen entzogen sind.

Faktor e beschreibt dagegen das Bündel jener Steuerungsinstrumente, die im Zuge des Wandels zur Outputorientierung in die Schulsysteme eingeführt wurden. Weniger leicht können die beiden weiteren Faktoren zugeordnet werden: Faktor c Schulpartner-Kooperationen umfasst evidenzbasierte und outputorientierte Überzeugungen, die die Rückmeldungen von Eltern und Schüler/innen in die Entscheidungsfindung einbinden wollen; darüber enthält er auch Items zu Kooperationen mit anderen sozialen Akteuren in der Umgebung der Schule (z. B. Gemeinde, andere Schulen). Faktor d Einzelschulische Qualitätsinstrumente scheint primär die Idee der Einzelschule als Handlungseinheit und Energiezentrum von Schulentwicklung einzufangen, wie sie in den Phasen 1 und 2 der Governance-Modernisierung propagiert wurde. Zugleich drückt möglicherweise gerade die Akzeptanz der Verpflichtung auf Instrumente wie Schulprogramme oder Selbstevaluationen bzw. auf regelmäßige Leistungsvereinbarungen mit der Schulaufsicht die Bereitschaft aus, sich einem bürokratisch-hierarchischen Steuerungsregime zu unterwerfen - oder eben die Konsequenzen einer outputorientierten Steuerung auf einzelschulischer Ebene umzusetzen. 


\section{Forschungsfragen}

Uns interessiert nun, welche steuerungsbezogenen Einstellungen bei Schulleitungen beobachtbar sind und ob jene Leitungspersonen, die den durch die GovernanceModernisierung propagierten Steuerungsvorstellungen anhängen, auch aktiver bei Schulentwicklung sind und ein outputorientiertes Leitungskonzept in ihrer alltäglichen Tätigkeit vertreten. Wir gehen dabei in folgenden Schritten vor:

Aus früheren Untersuchungen (Altrichter et al. 2012b) wissen wir, dass verschiedene Steuerungsstrategien von Schulleitungen nicht unbedingt wechselseitig exklusiv sind. Wir fragen zunächst in explorativer Absicht:

1. Gibt es Gruppen von Schulleiter/innen, die sich in Hinblick auf ihre governancebezogenen Einstellungen unterscheiden?

Basierend auf der Forschung zur Nutzung von Evidenzen ist es plausibel, anzunehmen, dass sich zumindest eine Gruppe identifizieren lässt, die Outputorientierung und Evidenzbasierung als Schulentwicklungsstrategien positiv gegenübersteht.

Im nächsten Schritt stellt sich die Frage, ob sich die gefundenen Gruppen hinsichtlich ihrer Schulentwicklungsaktivitäten unterscheiden. Eine zentrale Annahme des Wirkungsmodells neuer Steuerung besteht darin, dass Outputorientierung und Evidenzbasierung günstig für die Entwicklungsfähigkeit des Schulwesens sind (vgl. Analysegesichtspunkt 1 in Abschn. 1.1). Damit sich diese Behauptung realisieren kann, müssten jene Akteure, die Konzepte evidenzbasierter Steuerung schätzen, besonders aktiv bei Schulentwicklung sein.

2. Ist jene Gruppe von Schulleitungen, die durch eine hohe Wertschätzung von Outputorientierung charakterisiert ist, aktiver in Hinblick auf Schulentwicklung?

Der beschriebene Wandel in der Steuerung der Bildungssysteme hat auch - so wird in der Steuerungsliteratur geschlussfolgert - zu einer Veränderung der Anforderungen an Schulleitungen, ihrer Rollenvorstellungen und Verantwortlichkeiten geführt (vgl. Analysegesichtspunkt 2 in Abschn. 1.1 und 1.2): „Moderne outputorientierte Schulleitung“ beschäftigt sich weniger mit administrativen Aufgaben und direktem Unterrichten, sondern mehr mit evaluativen Aufgaben, Qualitätsmanagement, Schulentwicklung, Mitarbeiterführung, Außenkontakten und -darstellung.

3. Unterscheidet sich jene Gruppe von Schulleitungen, die durch eine hohe Wertschätzung von Outputorientierung charakterisiert ist, in ihren Führungsvorstellungen von den anderen Gruppen?

Wenn dies der Fall ist, so würden Proponent/innen evidenzbasierter Steuerung hoffen, dass sich solche Schulleitungen häufiger (als Schulleitungen mit anderen Steuerungspräferenzen) bei jenen Tätigkeiten engagieren, die ihnen Informationen über tatsächliche Unterrichtsprozesse, deren Wahrnehmungen durch Bezugspersonen sowie deren Ergebnisse versprechen, und zwar bei Unterrichtsbeobachtungen, Kommunikation mit Schüler/innen und Selbstevaluierung. Sie suchen das Gespräch mit Lehrpersonen über die pädagogische Arbeit statt diese allein den Lehrpersonen zu überantworten. Sie setzen klare Prioritäten und verstehen sich eher als Manager und Leiter/innen eines pädagogischen Betriebs denn als Unterrichtende mit Zusatzaufgaben. 


\section{Methodisches Vorgehen}

Beschreibung des Datenmaterials Zur Analyse dieser Fragestellungen nutzen wir Daten aus dem EU-geförderten Projekt „Impact of School Inspections on Teaching and Learning“ (Ehren et al. 2013). In dessen Rahmen wurden alle Schulleitungen aus Allgemeinbildenden Pflichtschulen (i.e. Primar- und Neue Mittelschulen, nicht aber höhere Schulen/Gymnasien) im österreichischen Bundesland Steiermark befragt. Die Datenerhebung fand online im Zeitraum von November 2012 bis Februar 2013 statt. In Summe wurden 678 Schulleiter/innen kontaktiert, von denen 362 den Fragebogen beantworteten. Etwa zwei Drittel der Schulleitungen in unserer Studie sind weiblich, die durchschnittliche Berufserfahrung beträgt sieben Jahre (SD: 6 Jahre). Die Mehrheit der Teilnehmer/innen leitet eine Volksschule (72\%). Die Stichprobe stimmt hinsichtlich Schulgröße, Anteil weiblicher Schulleitungen, Schüler- und Lehrerzahl mit der Gesamtpopulation im Bundesland überein (vgl. Altrichter et al. 2012c; Statistik Austria 2016; Vogtenhuber et al. 2012).

Einstellung zu Steuerungsstrategien Diese wurden mit einem mehrfach erprobten Instrument erhoben. Der einleitende Fragetext lautete: „Um die Qualität des Schulwesens weiterzuentwickeln, werden in verschiedenen Ländern neue Steuerungsinstrumente eingesetzt. Für wie geeignet halten Sie die folgenden Instrumente für die Entwicklung der Qualität des österreichischen Schulwesens?" Anschließend wurde eine Liste von 22 Steuerungsinstrumenten und -strategien angefügt. Als Antwortoptionen standen die Ausprägungen ,,völlig geeignet“ (=4), ,eher geeignet“ (=3), ,eher nicht geeignet“ (=2) und ,überhaupt nicht geeignet“" (=1) zur Auswahl (vgl. Kemethofer und Altrichter 2017).

Aktivitäten der Schulentwicklung Schulentwicklungsaktivitäten wurden durch die Zeit, die eine Schule im Vergleich zum Vorjahr für spezifische Entwicklungsmaßnahmen verwendet hat, operationalisiert. Die Schulleitungen sollten die Entwicklungsaktivitäten ihrer Schule auf einer fünfstufigen Skala mit den Ausprägungen „,deutlich mehr“ (=5) bis ,deutlich weniger“ $(=1)$ bewerten. Inhaltlich wurden drei Dimensionen unterschieden: Die Skala Schuleffektivität fokussiert auf Leitungsaktivitäten, die Unterrichtsentwicklung fördern sollen und bestand aus sechs Items (Bsp.: Beobachtung der Lernfortschritte aller Schüler/innen; $\alpha=0,82 ; M=3,60 ; S D=0,77$ ). Entwicklungskapazität umfasst Items, die nach kollegialer Zusammenarbeit und Abstimmung der schulischen Alltags- und Entwicklungstätigkeit fragen. Die Skala umfasst acht Items (Bsp.: Diskussion neuer Unterrichtsmethoden; $\alpha=0,85 ; M=4,31$; $S D=0,44)$. Items zur Selbstevaluierung fragen danach, wie viel Zeit in Prozesse der Evaluation, der Verbesserung und der Beteiligung relevanter Akteure investiert wurde. Die Skala bestand aus drei Items (Bsp.: Zeit für die Verbesserung des Selbstevaluierungsprozesses; $\alpha=0,89 ; M=3,63$; $S D=0,51$ ). Diese Konstrukte wurden bereits mehrfach international verwendet; eine ausführliche Beschreibung ist bei Gustafsson et al. (2015) zu finden.

Schulleitungstätigkeiten Das Tätigkeitsprofil der Schulleitungen wurde mittels des prozentualen Anteils unterschiedlicher Tätigkeiten an der Arbeitszeit abgebildet. 
Die Schulleiter/innen wurden dabei aufgefordert, den durchschnittlichen Prozentanteil verschiedener Tätigkeiten an ihrer Arbeitszeit einzugeben. Als Antwortoptionen standen „Administrative Aufgaben“ ( $M=35,08 ; S D=16,75)$, „Unterrichten, inklusive der Vertretung des Unterrichts anderer Lehrpersonen“ $(M=28,55 ; S D=22,27)$, „Diskussionen über erzieherische und unterrichtliche Fragen mit Lehrpersonen“ $(M=11,75 ; S D=7,09)$, ,Unterrichtsbeobachtungen“ $(M=6,04 ; S D=4,00)$, „,Kommunikation mit Schüler/innen außerhalb des Unterrichts bzw. Beschäftigung mit Schülerverhalten“ $(M=8,26 ; S D=6,37)$, ,Selbstevaluierung und Qualitätssicherung der Schule“ $(M=6,79 ; S D=4,15)$ und ,Sonstiges“ $(M=3,53 ; S D=4,78)$ zur Verfügung.

Statistische Analysen Die Beantwortung unserer Forschungsfragen erfolgt schrittweise: Zunächst nutzten wir eine Clusteranalyse mit k-Means-Algorithmus (vgl. Hastie et al. 2009) zur Gruppenbildung. Die Clusteranalyse ist ein Verfahren zur Konstruktion von Typologien, wobei Einheiten innerhalb eines Clusters deutlich größere Ähnlichkeit untereinander aufweisen als zwischen verschiedenen Clustern (Wiedenbeck und Züll 2010). Der k-Means-Algorithmus arbeitet dabei mit einer vorab bestimmten Anzahl von zu bildenden Gruppen. Die Clusteranlyse wurde zunächst für Lösungen mit 2-5 Gruppen berechnet. Daraus wurde die Variante mit 3 Gruppen gewählt, da hier die Kombination von Varianz zwischen den Gruppen und plausibler Interpretierbarkeit der ausgewiesenen Cluster optimal war.

Anschließend vergleichen wir die identifizierten Gruppen mittels Varianzanalysen. Die Effektstärke Omega-Quadrat $\left(\omega^{2}\right)$ dient zur Einschätzung der praktischen Bedeutsamkeit (Fritz et al. 2011). Für paarweise Gruppenunterschiede wird zudem die Effektstärke $d$ berichtet. Die Interpretation orientiert sich an den von Cohen (1988) genannten Werten. Ein Effekt von $\omega^{2}>0,01$ ist als schwach, $\omega^{2}>0,06$ als moderat und $\omega^{2}>0,14$ als stark einzustufen. Für $d$ gilt $d=0,20$ als schwach, $d=0,50$ als moderat und $d=0,80$ als stark. Die Berechnungen wurden mit den Statistikprogrammen R (R Core Team 2018), SPSS 22 und G*Power (Faul et al. 2007) durchgeführt.

Limitationen Aus methodischer Sicht ist darauf hinzuweisen, dass bei der Erhebung von Entwicklungsaktivitäten nicht der aktuelle Stand der Entwicklungsbemühungen, sondern die zeitlichen Veränderungen im Vergleich zum vorangegangenen Schuljahr erfasst wurden. Ebenfalls zu berücksichtigen ist, dass sich die Einleitungsfrage der Itembatterie zur Einschätzung von Steuerungsstrategien auf das Entwicklungspotential für das gesamte Schulwesen und nicht auf jenes für die Einzelschule bezieht. Eine Post-hoc Analyse zur Berechnung der Teststärke hat darüber hinaus ergeben, dass die Studie für schwache Effekte eine zu geringe Power aufweist. Die Chancen, dass bestehende Gruppenunterschiede auch tatsächlich als signifikant identifiziert werden, waren folglich eingeschränkt.

\section{Ergebnisse}

Governancebezogene Einstellungen In Hinblick auf Forschungsfrage 1 konnten mittels Clusteranalyse drei Gruppen von Schulleitungen mit unterschiedlichen 
Mustern von governancebezogenen Einstellungen identifiziert werden. Die Antwortmuster der Gruppen sind in Abb. 1 dargestellt. Auf der x-Achse unten werden die Einzelitems (Steuerungsinstrumente und -strategien) genannt, auf der x-Achse oben ist die Zuordnung der Einzelitems zu den in Abschn. 1.3 genannten Einstellungsfaktoren sichtbar.

Eine Gruppe von Schulleitungen attribuiert allen Ansätzen zur Systemsteuerung hohes Potential zur Qualitätsentwicklung im Schulwesen, und zwar auch höheres Potential als die beiden anderen Gruppen mit Ausnahme des Faktors „Ressourcensteigerung". Am deutlichsten unterscheidet sich dieser Typ von Schulleiter/in, indem er auch evidenzbasierten Steuerungsinstrumenten ein gewisses Potential zugesteht, was bei den anderen Gruppen deutlich weniger der Fall ist. Auch in Ansätzen einzelschulischer Qualitätsentwicklung und in Kooperationen mit Schulpartner/innen sieht diese Gruppe hohes Potential zur Qualitätsentwicklung im Schulwesen. Insgesamt konnten 27,5\% der Schulleiter/innen in unserem Sample diesem Typ zugeordnet werden, den wir als Typ 1 Evidenzbasierte Schulentwicklung bezeichnen.

Eine zweite Gruppe von Schulleiter/innen unterscheidet sich von allen anderen dadurch, dass sie mehr Ressourcen für Schulen und Klassen als geeignetstes Instrument der Qualitätsentwicklung ansieht. Hohes Potential wird auch der Ausbildung von Lehr- und Leitungspersonal zugeschrieben. Ansätze, die Schulentwicklung durch Datenfeedback forcieren, werden im Vergleich zu den (eher) inputorientierten Strategien skeptischer betrachtet. Dieses Cluster - Typ 2 Entwicklung durch Ressourcen - umfasst 37,5\% der Schulleitungen.

Eine dritte Gruppe gleicht in ihrem Profil weithin dem Typ 2, außer in ihrer Einschätzung von Ressourcensteigerung, der sie geringeren Wert für die Qualitätsentwicklung zugesteht als die beiden anderen Typen. Dadurch liegen die meisten Einschätzungen auf einem mittleren (unentschiedenem?) Niveau - mit zwei Ausnahmen: Ihre Skepsis gegenüber ,internationalen Vergleichsstudien“ und „Nationalen Bildungsberichten" ist deutlich ausgeprägt; am ehesten wird unterrichtsbezogenen Ansätzen (wie ,weniger Schüler/innen in den Klassen“, „,bessere Ausbildung der Lehrer/innen“ und „mehr Praxiserfahrung für neue/junge Lehrer/innen“) erhöhtes Potential für Qualitätsentwicklung attestiert. Typ 3 wird hier - in Ermangelung einer sprechenderen Benennung - als weniger entwicklungsorientiert bezeichnet und umfasst einen Anteil von 35\% der Schulleitungen unserer Studie.

Die Varianz zwischen den in der Clusteranalyse identifizierten Gruppen (IntraKlassen-Korrelation, ICC) macht den diskriminierenden Einfluss der einzelnen Items auf die Clusterbildung sichtbar. Die ICC-Werte liegen im Bereich zwischen 0,41 und 0,09, wobei jene Items, die die Strategie Ressourcensteigerung erfassen, tendenziell die höchste Varianz zwischen den Clustern aufweisen (vgl. Abb. 1).

Die Gruppen unterscheiden sich auch in Hinblick auf die Schulgröße und die Berufserfahrung der Schulleitung. ,Evidenzbasierte“ Schulleiter/innen sind signifikant häufiger in größeren Schulen zu finden als Schulleitungen in der weniger entwicklungsorientierten Gruppe $\left(F=4,54_{2}, 273 ; p=0,012\right)$. Schulleiter/innen mit längerer Berufserfahrung sind häufiger in der weniger entwicklungsorientierten Gruppe zu finden $\left(F=3,98_{2}, 305 ; p=0,020\right)$. Keine statistisch signifikanten Unterschiede zeigen sich bei Schultyp und Geschlecht der Schulleitung (vgl. Kemethofer und Altrichter 2017). 
Tab. 1 Gruppenvergleich für Aktivitäten der Schulentwicklung

\begin{tabular}{lllllll}
\hline & Evidenz & Ressourcen & $\begin{array}{l}\text { Weniger } \\
\text { Entwick- } \\
\text { lung }\end{array}$ & Teststatistik & \\
& & & & & \\
& $M(S E)$ & $M(S E)$ & $M(S E)$ & $F_{d f}$ & $p$ & $\omega^{2}$ \\
\hline $\begin{array}{l}\text { Entwicklungsaktivität } \\
\text { Selbstevaluierung }\end{array}$ & $3,79(0,09)_{\mathrm{a}}$ & $3,63(0,08)_{\mathrm{a}}$ & $3,54(0,07)_{\mathrm{b}}$ & $2,442,285$ & 0,089 & 0,01 \\
Schuleffektivität & $3,83(0,06)_{\mathrm{a}}$ & $3,58(0,05)_{\mathrm{b}}$ & $3,56(0,05)_{\mathrm{b}}$ & $7,99,301$ & $<0,001$ & 0,04 \\
Entwicklungskapazität & $3,95(0,06)_{\mathrm{a}}$ & $3,79(0,06)_{\mathrm{b}}$ & $3,60(0,05)_{\mathrm{c}}$ & $9,662,291$ & $<0,001$ & 0,06 \\
\hline
\end{tabular}

Gruppen mit abweichendem tiefgestelltem Index $(a, b, c)$ unterscheiden sich im paarweisen Vergleich (t-Test) statistisch signifikant voneinander $(p \leq 0,05)$

Die drei Cluster sind in Hinblick auf die konkurrierenden Steuerungsvorstellungen gut zu interpretieren:

Typ 1 Evidenzbasierte Schulentwicklung enthält jene Schulleitungen, die die positivsten Einstellungen zu den Instrumenten der Outputorientierung oder evidenzbasierten Entwicklung haben. Für die Protagonist/innen der Governance-Modernisierung ist erfreulich, dass in dieser Gruppe auch jene Schulleitungen erfasst sind, die die Strategie der „Qualitätsentwicklung auf Ebene der Einzelschule“ am stärksten befürworten, d.h. die wahrscheinlich auch bereit sind, die Ergebnisse evidenzbasierter Rückmeldungen in Einzelschulentwicklung zu übersetzen (was in Frage 2 überprüft wird). Die ebenfalls überdurchschnittlichen Werte bzgl. der Kooperation mit Schulpartner/innen und Gemeinde zeichnen diese Gruppe möglicherweise als „dynamische Akteure“ in der Schullandschaft aus.

Typ 2 Entwicklung durch Ressourcen scheint dagegen für ,,inputorientierte Einstellungen“ zu stehen, die gerade durch die Modernisierungspolitik zurückgedrängt werden sollen. Mitglieder dieser Gruppe erwarten sich Qualitätsentwicklung vor allem durch den Einsatz materieller Ressourcen, die von Staat und Schulerhaltern zur Verfügung gestellt werden sollen (womit sie sich möglicherweise auch von eigener Verantwortung dispensieren). Bezüglich des Faktors „Mitarbeiter-Qualifizierung“, der als ,,inputorientiert“ (personelle Ressourcen) interpretiert werden kann, nimmt dieser Typ keine Spitzenwerte ein. Dies kann aber an der inhaltlichen Ausgestaltung des Faktors liegen: „Personelle Ressourcen“ werden durchgehend als wichtig und z. T. als ,gestaltbar“" (z.B. durch Fortbildung) angesehen.

Der weniger entwicklungsorientierte Typ 3 lässt sich auf der Dimension Inputvs. Outputorientierung nicht eindeutig verorten. Möglicherweise handelt es sich um Akteure, die vorsichtig wählen oder die in keine der angebotenen Strategien der Qualitätsentwicklung besondere Hoffnungen setzen.

Schulentwicklungsaktivität In Forschungsfrage 2 interessiert uns, ob jene, die Outputorientierung und Evidenzbasierung für produktive Strategien der Qualitätsentwicklung halten, auch tatsächlich mehr Entwicklungsaktivität zeigen. Tab. 1 weist die Mittelwerte der abhängigen Variablen sowie die Kennwerte zur Prüfung der Mittelwertunterschiede und Effektstärken aus. Der $F$-Test ergab einen statistisch signifikanten Unterschied für zwei der drei untersuchten Merkmale. 
Tab. 2 Gruppenvergleich für den Zeitaufwand unterschiedlicher Tätigkeiten als Schulleiter/in

\begin{tabular}{|c|c|c|c|c|c|c|}
\hline & Evidenz & Ressourcen & Weniger & Teststatistik & & \\
\hline & $\%$ & $\%$ & $\%$ & $F_{d f}$ & $p$ & $\omega^{2}$ \\
\hline \multicolumn{7}{|l|}{ Tätigkeiten } \\
\hline $\begin{array}{l}\text { Administrative Tätig- } \\
\text { keiten }\end{array}$ & $38,58 \mathrm{a}$ & $35,70 \mathrm{a}$ & $32,98 \mathrm{a}$ & $2,732,299$ & 0,067 & 0,01 \\
\hline Unterrichten & $19,87 \mathrm{a}$ & $29,11_{b}$ & $33,88_{b}$ & $10,51_{2,299}$ & $<0,001$ & 0,06 \\
\hline $\begin{array}{l}\text { Diskussionen über } \\
\text { erzieherische und unter- } \\
\text { richtliche Fragen }\end{array}$ & $13,67 \mathrm{a}$ & $11,19 \mathrm{~b}$ & $11,95 \mathrm{a}, \mathrm{b}$ & $3,382,299$ & 0,035 & 0,02 \\
\hline Unterrichtsbeobachtungen & $7,29 \mathrm{a}$ & $5,33 b$ & $5,57 \mathrm{~b}$ & $6,682,300$ & 0,001 & 0,04 \\
\hline $\begin{array}{l}\text { Kommunikation mit } \\
\text { Schüler/innen }\end{array}$ & $9,19 \mathrm{a}$ & $8,23 \mathrm{a}$ & $7,13_{a}$ & $2,672,299$ & 0,071 & 0,01 \\
\hline $\begin{array}{l}\text { Selbstevaluierung/ } \\
\text { Qualitätssicherung der } \\
\text { Schule }\end{array}$ & $7,68 \mathrm{a}$ & $6,70_{a}, \mathrm{~b}$ & $6,13 \mathrm{~b}$ & $3,402,299$ & 0,035 & 0,02 \\
\hline Sonstiges & $3,73_{\mathrm{a}}$ & $2,92_{\mathrm{a}}$ & $3,74 a$ & $1,182,299$ & 0,308 & 0,00 \\
\hline
\end{tabular}

Gruppen mit abweichendem tiefgestellten Index $(\mathrm{a}, \mathrm{b})$ unterscheiden sich im paarweisen Vergleich (t-Test) statistisch signifikant voneinander $(p \leq 0,05)$

Dabei berichten Schulleiter/innen der Gruppe Evidenzbasierte Schulentwicklung bei allen einbezogenen Maßen über vergleichsweise mehr Entwicklungsaktivitäten als die anderen beiden Gruppen, allerdings erreichen die Mittelwertsunterschiede nicht immer die Signifikanzgrenze (vgl. Tab. 1). Die Unterschiede zwischen den Gruppen der Evidenz- (Typ 1) und der Ressourcenorientierung (Typ 2) verlaufen auch nicht so klar, wie dies von einer outputorientierten Politikvorstellung vorhergesagt würde. Evidenzbasierte Schulleitungen unterscheiden sich signifikant von ressourcenorientierten, indem sie mehr Aktivitäten zur Förderung von Unterrichtsentwicklung (Schuleffektivität) $(d=0,48)$ und von kollegialer Zusammenarbeit (Entwicklungskapazität) setzen $(d=0,31)$, nicht jedoch zur Förderung von Selbstevaluierung. Die weniger entwicklungsorientierte Gruppe (Typ 3) hebt sich dagegen sowohl von der evidenz- als auch von der ressourcenorientierten Gruppe durch signifikant geringe Werte bei Aktivitäten zur Selbstevaluierung $(d=0,31$ bzw. $d=0,10)$ sowie zur Verbesserung der Entwicklungskapazität $(d=0,62$ bzw. $d=0,35)$ ab. Die praktische Bedeutsamkeit der gefundenen Unterschiede $\left(\omega^{2}\right.$ und $\left.d\right)$ ist als schwach bis moderat zu bewerten.

Tätigkeitsprofil Der beschriebene Governancewandel hat auch zu veränderten Arbeitsanforderungen für Schulleitung geführt. Entsprechend Frage 3 wird in Tab. 2 das Zeitkontingent der identifizierten Typen für Leitungsaufgaben dargestellt. Der Löwenanteil der verfügbaren Zeit fällt in allen drei Gruppen auf administrative Aufgaben und Unterrichten, wobei der evidenzbasierte Typ signifikant weniger Zeit in einer Klasse verbringt. Die evidenzorientierte Gruppe engagiert sich indes häufiger bei jenen Tätigkeiten, die ihnen ein klareres Bild der tatsächlichen Unterrichtsprozesse liefern sowie Erkenntnisse über dessen Ergebnisse versprechen, bspw. aus 
einer beobachtenden Perspektive oder durch Wahrnehmungen von Bezugspersonen. Diese Schulleiter/innen investieren einen höheren Anteil ihrer Arbeitszeit in Unterrichtsbeobachtungen (signifikant mehr als Typ $2(d=0,47)$ und $3(d=41))$ sowie Selbstevaluierung und Qualitätssicherung (signifikant mehr als Typ $3(d=0,39)$ ). Sie suchen auch häufiger das Gespräch mit Lehrpersonen über die pädagogische Arbeit (signifikant mehr als Typ $2(d=0,36))$ statt diese allein den Lehrkräften zu überantworten (vgl. Kronsfoth et al. 2018). Insgesamt scheinen sie sich eher als Manager/ innen und Leiter/innen eines pädagogischen Betriebs denn als Unterrichtende mit Zusatzaufgaben zu verstehen.

\section{Diskussion, Limitationen und Ausblick}

In diesem Beitrag wurde untersucht, ob sich die bildungspolitisch gewollten, neuen outputorientierten Konzepte in den Steuerungsvorstellungen österreichischer Schulleitungen wiederfinden und welche Zusammenhänge zu anderen Tätigkeitsmerkmalen, insbesondere Schulentwicklungs- und Leitungsaktivitäten, bestehen. Die Ergebnisse zeigen, dass es tatsächlich eine Gruppe von Schulleitungen gibt, die Instrumente der Outputorientierung und Evidenzbasierung am relativ besten einschätzt und die sich in einer Clusteranalyse deutlich von einer ressourcenorientierten Gruppe und einer anderen, weniger entwicklungsorientierten, die weder Ressourcen- noch Outputorientierung bevorzugt, unterscheidet.

Entsprechend der bildungspolitischen Idee, dass neue Systemsteuerung auch auf Einzelschulebene aktiv evaluierende und entwickelnde Akteure benötigt, sind systematisch mehr Schulentwicklungsaktivitäten bei der outputorientierten Gruppe zu erwarten. Diese bildungspolitische Vorhersage tritt aber in unserem Sample nicht durchgehend ein. Outputorientierte Leitungen berichten zwar über signifikant mehr Aktivitäten zur Förderung von Unterrichtsentwicklung als die beiden anderen Gruppen. In Hinblick auf die kollegiumsbezogene Kapazität, Entwicklungsprozesse durchführen zu können, und auf Selbstevaluierungsmaßnahmen ist der Unterschied lediglich zur weniger entwicklungsorientierten Gruppe signifikant. Das letztere Ergebnis ist vielleicht dadurch erklärbar, dass im Jahr der Erhebung das neue Qualitätsmanagementsystem SQA propagiert wurde, wodurch möglicherweise die Aufmerksamkeit aller Leitungspersonen auf Selbstevaluierung gelegt wurde.

Outputorientierte Schulleitungen scheinen ihre Energien tatsächlich so auf verschiedene Tätigkeiten zu verteilen, wie es von den bildungspolitischen Vorstellungen zur evidenzbasierten Steuerung nahegelegt wird. Sie berichten, weniger Zeit für Unterrichten und mehr für Unterrichtsbeobachtungen und Gespräche mit den Lehrpersonen über erzieherische und unterrichtliche Fragen zu verwenden als die ressourcenorientierte Gruppe. Wiederum findet sich jedoch kein Unterschied zwischen den Gruppen bzgl. Selbstevaluierung, aber auch bzgl. administrativer Aufgaben, die vielleicht einen real weniger disponierbaren Kern bei allen Leitungskonzepten ausmachen.

Bezogen auf unsere theoretischen Ausgangsüberlegungen kann man konstatieren, dass die vorliegenden Daten zum größten Teil mit den Annahmen übereinstimmen. Im Zuge der Modernisierung der Governance des Bildungssystem sind zwei Grup- 
pen von Schulleitungspersonen deutlich zu unterscheiden: Eine outputorientierte Gruppe, die den Neuerungsmaßnahmen Qualitätspotential zuerkennt, ihre Tätigkeit im Sinne neuer Leistungsanforderungen umgestaltet hat und auch mehr Entwicklungsaktivitäten berichtet. Erklärungsbedürftig bleiben die fehlenden Unterschiede bei Selbstevaluierungsmaßnahmen. In einer Entwicklungsperspektive wäre es interessant herauszufinden, ob die Gruppe der evidenzbasierten Leitungspersonen nur durch „Konversion“ der ohnehin entwicklungsaktiven Leitungen entstanden ist sowie ob (und wodurch) sich diese durch die Erfahrung mit neuen Steuerungsstrategien vergrößert oder nicht (worauf Interviews mit der Schulaufsicht hindeuten; vgl. Kemethofer und Wiesner 2016). Weiters wäre es wichtig, die dritte gefundene Gruppe, die keine der genannten Strategien bevorzugt, genauer in ihren Vorstellungen, Arbeitsbedingungen und tatsächlichen Tätigkeiten zu beschreiben. Gruppe 2 und 3, die hier - entsprechend der Fragerichtung der Studie - vor allem durch ihre Skepsis zur Modernisierungsstrategie „Outputorientierung“ charakterisiert wurden, müssten auch besser in ihren positiven Motivationen (die z. B. in der Wahrung der Autonomie, Kosten-Nutzen-Abschätzungen, Betonung von interner Evaluation usw. liegen könnten) beschrieben und verstanden werden.

Aus bildungspolitischer Perspektive ist es für die Protagonist/innen evidenzbasierter Entwicklung erfreulich, dass es offenbar gelungen ist, einen Teil der Schulleitungen für die Leitideen der Modernisierungspolitik zu gewinnen. Da sich diese Leitungspersonen gleichzeitig überdurchschnittlich stark für einzelschulische Qualitätsentwicklung und Kommunikation mit den Schulpartnern aussprechen und auch ihre Tätigkeit eher entwicklungsorientiert gestalten, kann man annehmen, dass gerade die entwicklungsbereiten und -aktiven Teile der Zielgruppe angesprochen werden konnten. Unerfreulich ist aus dieser Perspektive aber, dass nationale Bildungsberichte und internationale Vergleichsstudien - beides Instrumente einer evidenzbasierten Steuerung - vergleichsweise geringe Zustimmungsraten aufweisen. Mit Blick auf die eigene Schule dürften sowohl Bildungsberichte als auch internationale Studien jedoch vergleichsweise wenig Entwicklungspotential aufweisen und so die Zurückhaltung der Schulleitungen erklären. Die anderen beiden Instrumente evidenzbasierter Steuerung, Bildungsstandards und Standardüberprüfungen, beziehen sich stärker auf die schulische Arbeit und weisen Zustimmungsraten auf, die im Bereich der Instrumente einzelschulischer Qualitätsentwicklung und Schulpartner-Kooperation liegen.

Welche praktischen Schlüsse kann man daraus für die weitere Entwicklung ziehen? Einesteils ist der Unterschied zwischen den genannten Gruppen vielleicht nicht so schroff, wie es in dieser Gegenüberstellung erscheint: Nur in zwei (allerdings deutlich ,evidenzbasierten“) Items liegen Leitungspersonen vom Typ 2 und 3 unter dem theoretischen Mittelwert; es gibt also wahrscheinlich deutliche Übereinstimmung in Hinblick auf sinnvolle Entwicklungsstrategien, wenn auch unterschiedlich enthusiastische Zustimmung. Das eröffnet ein Feld für Bewusstseinsbildung und Kompetenzaufbau für evidenzbasierte Arbeitsgestaltung in Leitungsfortbildungen und Dienstbesprechungen. Gerade die „Bilanz- und Zielvereinbarungsgespräche“ 
im Zuge des neuen Qualitätsmanagementsystems SQA² könnten die Möglichkeit bieten, den Schulleitungen Denk- und Arbeitsweisen sowie praktische Konsequenzen einer evidenzbasierten Entwicklung nahezubringen, wenn die Schulaufsicht über entsprechende Kompetenzen verfügt und die Gespräche in diesem Sinne gestaltet.

Bei der Auswahl von neuen Schulleitungen besteht in einer Zeit, in der weniger Personen diese Stellen anstreben, wohl wenig Handlungsspielraum. Bei einer eventuellen Werbung für solche Positionen und bei der informellen „Entdeckung von Schulleitungstalenten“" sollten aber neue Tätigkeitsgesichtspunkte deutlich kommuniziert werden. Besonderes Augenmerk verdient die Schulleiterausbildung, die in Österreich gegenwärtig reformiert wird (Huber 2015; Kanape und Kemethofer 2017; Nusche et al. 2016). Sowohl in der Grundausbildung, die vor der Bestellung zur Leitungsperson erfolgt, als auch in der Begleitung beginnender Direktor/innen und in nachgelagerten Ausbildungsteilen sollten Konzepte und Kompetenzen zu einer veränderten Leitungstätigkeit eine wichtige Rolle spielen. Für Maßnahmen in allen angesprochenen Bereich wäre es hilfreich, wenn klare professionelle Standards für Schulleitung (vgl. NPBEA 2015) Orientierung und Bezugspunkte für die Arbeitsgestaltung bieten würden.

Abschließend ist noch auf die Limitationen der Studie hinzuweisen, die bei der Interpretation der Befunde zu beachten sind: Zunächst ist anzumerken, dass sich seit dem Erhebungszeitpunkt bildungspolitisch viel getan hat. So wurden bspw. Unterstützungssysteme für Schulen im Umgang mit Datenfeedback eingerichtet (z.B. Rückmeldemoderation, Handbücher) und Angebote entwickelt, die für die Implementierung neuer Steuerungsinstrumente auf Schulebene förderlich sein können (z.B. Qualifizierungsmaßnahmen). Mit der Initiative SQA wurde darüber hinaus versucht, die Verbindlichkeit evidenzorientierter Schulentwicklung zu erhöhen Der Mehrwert der Studie liegt trotz der Einschränkungen (die schon in Abschn. 3 dargelegt wurden) darin, erstmals aufzuzeigen, dass es - bezogen auf aktuell diskutierte Steuerungsansätze - unterschiedliche Typen von Schulleitungen gibt, die sich auch durch ihr schulisches Handeln unterscheiden.

Funding Open access funding provided by Johannes Kepler University Linz.

Open Access Dieser Artikel wird unter der Creative Commons Namensnennung 4.0 International Lizenz (http://creativecommons.org/licenses/by/4.0/deed.de) veröffentlicht, welche die Nutzung, Vervielfältigung, Bearbeitung, Verbreitung und Wiedergabe in jeglichem Medium und Format erlaubt, sofern Sie den/die ursprünglichen Autor(en) und die Quelle ordnungsgemäß nennen, einen Link zur Creative Commons Lizenz beifügen und angeben, ob Änderungen vorgenommen wurden.

\section{Literatur}

Ajzen, I. (1991). The theory of planned behavior. Organizational Behavior and Human Decision Processes, 50(2), 179-211.

Altrichter, H. (2015). Governance - Steuerung und Handlungskoordination bei der Transformation von Bildungssystemen. In H. J. Abs, T. Brüsemeister, M. Schemmann \& J. Wissinger (Hrsg.), Governance im Bildungssystem (S. 21-63). Wiesbaden: Springer VS.

Altrichter, H. (2017). Von der Schulinspektion zum systemischen Qualitätsmanagement. Zeitschrift für Bildungsverwaltung, 33(2), 27-41.

\footnotetext{
2 Diese entsprechen in anderen Schulsystemen Zielvereinbarungen zwischen Schulleitung und Schulauf-
} sicht. 
Altrichter, H., \& Kemethofer, D. (2015). Neue Ansätze der Steuerung des Schulsystems und die Einstellung von Schulleitungen. Bildung und Erziehung, 68(3), 291-310.

Altrichter, H., \& Merki, M. K. (Hrsg.). (2016). Handbuch Neue Steuerung im Schulsystem. Wiesbaden: Springer VS.

Altrichter, H., Brüsemeister, T., \& Heinrich, M. (2005). Merkmale und Fragen einer Governance-Reform am Beispiel des österreichischen Schulwesens. Österreichische Zeitschrift für Soziologie, 30(4), 6-28.

Altrichter, H., Kemethofer, D., \& Leitgöb, H. (2012a). Ansätze der Systemsteuerung in der Einschätzung von Schulleiterinnen und Schulleitern. Empirische Pädagogik, 26(1), 12-32.

Altrichter, H., Kemethofer, D., \& Leitgöb, H. (2012b). Schulentwicklung und Systemsteuerung. In F. Eder (Hrsg.), PISA 2009. Nationale Zusatzanalysen für Österreich (S. 228-253). Münster: Waxmann.

Altrichter, H., Kemethofer, D., \& Schmidinger, E. (2012c). Teaminspektion in der Steiermark. Ergebnisse der ersten Befragung im Rahmen des ISI-TL-Projekts. http://schoolinspections.eu/impact/ teaminspektion-in-der-steiermark-in-german/. Zugegriffen: 28. Aug. 2018.

Ball, S., Maguire, M., \& Braun, A. (2012). How schools do policy. Oxon: Routledge.

Bellmann, J. (2016). Datengetrieben und/oder evidenzbasiert? Zeitschrift für Erziehungswissenschaft, 19(1), 147-161.

Brauckmann, S., \& Herrmann, C. (2012). Schulleitungshandeln im Rahmen Neuer Steuerung: Belastung oder Chance? Zeitschrift für Bildungsverwaltung, 28(1), 87-98.

Brauckmann, S., \& Pashiardis, P. (2012). Contextual framing for school leadership training. Journal of Management Development, 31(1), 18-33.

Brauckmann, S., \& Pashiardis, P. (2016). European perspectives. The policy making and shaping of high quality leadership in school systems across Europe-expectation setting. In P. Pashiardis \& O. Johansson (Hrsg.), Successful school leadership. International perspectives (S. 179-191). London: Bloomsbury.

Brauckmann, S., \& Schwarz, J. (2015). No time to manage? The trade-off between relevant tasks and actual priorities of school leaders in Germany. International Journal of Educational Management, 29(6), 749-765.

Brown, C., \& Zhang, D. (2017). How can school leaders establish evidence-informed schools. Educational Management Administration \& Leadership, 45(3), 382-401.

Cohen, J. (1988). Statistical Power Analysis for the Behavioral Science. Hillsdale: Erlbaum.

Day, C., Gu, Q., \& Sammons, P. (2016). The impact of leadership on student outcomes. Educational Administration Quarterly, 52(2), 221-258.

Demski, D. (2017). Evidenzbasierte Schulentwicklung. Wiesbaden: Springer VS.

Earl, L., \& Fullan, M. (2003). Using data in leadership for learning. Cambridge Journal of Education, 33(3), 383-394.

Ehren, M.C.M., Altrichter, H., McNamara, G., \& O’Hara, J. (2013). Impact of school inspections on improvement of schools-describing assumptions on causal mechanisms in six European countries. Educational Assessment, Evaluation and Accountability, 25(1), 3-43.

Faul, F., Erdfelder, E., Lang, A. G., \& Buchner, A. (2007). G*power 3: a flexible statistical power analysis program for the social, behavioral, and biomedical sciences. Behavior research methods, 39(2), 175-191.

Fend, H. (1986). Gute Schulen - schlechte Schulen. Die einzelne Schule als pädagogische Handlungseinheit. Die Deutsche Schule, 82(3), 275-293.

Fend, H. (2006). Neue Theorie der Schule. Wiesbaden: VS.

Fend, H. (2011). Die Wirksamkeit der Neuen Steuerung - theoretische und methodische Probleme ihrer Evaluation. Zeitschrift für Bildungsforschung, 1(1), 5-24.

Fritz, C. O., Morris, P.E., \& Richler, J. J. (2011). Effect size estimates: current use, calculations, and interpretation. Journal of Experimental Psychology, 141(1), 2-18.

Groß Ophoff, J. (2013). Lernstandserhebungen: Reflexion und Nutzung. Münster: Waxmann.

Gustafsson, J.-E., Ehren, M.C.M., Conyngham, G., McNamara, G., Altrichter, H., \& O’Hara, J. (2015). From inspection to quality. Studies in Educational Evaluation, 47, 47-57.

Hallinger, P. (2011). Leadership for learning: lessons from 40 years of empirical research. Journal of Educational Administration, 49(2), 125-142.

Hastie, T., Tibshirani, R., \& Friedman, J. (2009). The elements of statistical learning. New York: Springer. Heid, H. (2003). Standardsetzung. Zeitschrift für Pädagogik, 49(47), 176-193.

Higham, R., \& Earley, P. (2013). School autonomy and government control. Educational Management Administration \& Leadership, 41(6), 701-717.

Huber, S. G. (Hrsg.). (2015). Schule gemeinsam gestalten - Entwicklung von Kompetenzen für pädagogische Führung. Münster: Waxmann. 
Huber, S. G., Wolfgramm, C., \& Kilic, S. (2013). Vorlieben und Belastungen im Schulleitungshandeln. In S. G. Huber (Hrsg.), Jahrbuch Schulleitung 2013 (S. 259-271). Köln: Carl Link.

Jornitz, S. (2008). Was bedeutet eigentlich „evidenzbasierte Bildungsforschung“? Die Deutsche Schule, $100(2), 206-216$.

Kanape, A., \& Kemethofer, D. (2017). Schulleitung in Österreich: Zwischen Steuerung und Autonomie. In A. Paseka, M. Heinrich, A. Kanape \& R. Langer (Hrsg.), Schulentwicklung zwischen Steuerung und Autonomie (S. 119-136). Münster: Waxmann.

Kemethofer, D., \& Altrichter, H. (2017). Der Beitrag neuer Steuerungsinstrumente zur Qualitätsentwicklung in der Einschätzung von Schulleitungen. In U. Steffens, K. Maag Merki \& H. Fend (Hrsg.), Schulgestaltung (S. 249-267). Münster: Waxmann.

Kemethofer, D., \& Wiesner, C. (2016). Die Nutzung der Bildungsstandards aus Perspektive der Schulaufsicht. In C. Fridrich, R. Klingler, R. Potzmann, W. Greller \& R. Petz (Hrsg.), Forschungsperspektiven 8 (S. 109-123). Wien: LIT.

Kronsfoth, K., Muslic, B., Graf, T., \& Kuper, H. (2018). Der Zusammenhang zwischen Führungsdimensionen in der Schulleitung und der Nutzung von Ergebnisrückmeldungen aus Vergleichsarbeiten. Die Deutsche Schule, 11(0), 47-64.

Kuper, H. (2008). Wissen - Evaluation - Evaluationswissen. In T. Brüsemeister \& K.-D. Eubel (Hrsg.), Evaluation, Wissen und Nichtwissen (S. 61-74). Wiesbaden: VS.

Martens, K., Nagel, A.-K., Windzio, M., \& Weymann, A. (2010). Transformation of education policy. Basingstoke: Palgrave.

Meyer, J. W., \& Rowan, B. (1977). Institutionalized organizations: formal structure as myth and ceremony. American Journal of Sociology, 83(2), 340-363.

Mintrop, R., \& Coghlan, E. (2018). Datenbasiertes Schulleitungshandeln - Befunde und praktische Erfahrungen aus den USA. Die Deutsche Schule, 110(1), 10-26.

NPBEA [National Policy Board for Educational Administration] (2015). Professional standards for educational leaders. Reston: NPBEA.

Nusche, D., Radinger, T., Busemeyer, M.R., \& Theisens, H. (2016). OECD reviews of school resources: Austria. Paris: OECD. https://doi.org/10.1787/9789264256729-en.

R Core Team (2018). R: A language and environment for statistical computing. Vienna: R Foundation for Statistical Computing.

Schimank, U. (2007). Die Governance-Perspektive: Analytisches Potenzial und anstehende konzeptionelle Fragen. In H. Altrichter, T. Brüsemeister \& J. Wissinger (Hrsg.), Educational Governance (S. 231-260). Wiesbaden: VS.

Schratz, M. (2016). Austria: overcoming a bureaucratic heritage as a trigger for research on leadership. In A.H. Ärlestig, C. Day \& O. Johansson (Hrsg.), A decade of research on school principals (S. 307-329). Cham: Springer.

Spillane, J.P. (2012). Data in practice: conceptualizing data-based decision-making phenomena. American Journal of Education, 118(2), 113-141.

Stamm, M. (2003). Evaluation und ihre Folgen für die Bildung. Münster: Waxmann.

Statistik Austria (2016). Bildung in Zahlen 2014/15. http://www.statistik.at/web_de/services/publikationen/ 5/index.html?id=5\&listid=5\&detail=461. Zugegriffen: 28. Aug. 2018.

Vogtenhuber, S., Lassnigg, L., Bruneforth, M., Herzog-Punzenberger, B., Auer, C., Gumpoldsberger, H., \& Schmich, J. (2012). Inputs - Personelle und finanzielle Ressourcen. In M. Bruneforth, B. Herzog-Punzenberger \& L. Lassnigg (Hrsg.), Das Schulsystem im Spiegel von Daten und Indikatoren. Nationaler Bildungsbericht Österreich 2012, (Bd. 1, S. 31-60). Graz: Leykam.

Wanberg, C.R., \& Banas, J. T. (2000). Predictors and outcomes of openness to changes in a reorganizing workplace. Journal of Applied Psychology, 85(1), 132-142.

Wiedenbeck, M., \& Züll, C. (2010). Clusteranalyse. In C. Wolff \& H. Best (Hrsg.), Handbuch der sozialwissenschaftlichen Datenanalyse (S. 525-552). Wiesbaden: VS.

Wiesner, C., Schreiner, C., Breit, S., Kemethofer, D., George, A. C., \& Angerer, S. (2016). Die Bedeutsamkeit der professionellen Reflexion und Rückmeldekultur für eine evidenzorientierte Schulentwicklung durch Bildungsstandardüberprüfungen. journal für schulentwicklung, 20(4), 18-26.

Wurster, S., \& Gärtner, H. (2013). Schulen im Umgang mit Schulinspektion und deren Ergebnissen. Zeitschrift für Pädagogik, 59(3), 425-445.

Zeitler, S., Asbrand, B., \& Heller, N. (2013). Steuerung durch Bildungsstandards - Bildungsstandards als Innovation zwischen Implementation und Rezeption. In M. Rürup \& I. Bormann (Hrsg.), Innovationen im Bildungswesen (S. 127-147). Wiesbaden: Springer.

Zukunftskommission, Eder, F., Specht, W., Spiel, C., \& Wimmer, M. (2005). Abschlussbericht der Zukunftskommission. Wien: BMBWK. 